\title{
Orbit Determination with the two-body Integrals
}

\author{
G. F. Gronchi, ${ }^{*}$ L. Dimare ${ }^{\dagger}$ A. Milani ${ }^{\ddagger}$
}

\begin{abstract}
We investigate a method to compute a finite set of preliminary orbits for solar system bodies using the first integrals of the Kepler problem. This method is thought for the applications to the modern sets of astrometric observations, where often the information contained in the observations allows only to compute, by interpolation, two angular positions of the observed body and their time derivatives at a given epoch; we call this set of data attributable. Given two attributables of the same body at two different epochs we can use the energy and angular momentum integrals of the two-body problem to write a system of polynomial equations for the topocentric distance and the radial velocity at the two epochs. We define two different algorithms for the computation of the solutions, based on different ways to perform elimination of variables and obtain a univariate polynomial. Moreover we use the redundancy of the data to test the hypothesis that two attributables belong to the same body (linkage problem). It is also possible to compute a covariance matrix, describing the uncertainty of the preliminary orbits which results from the observation error statistics. The performance of this method has been investigated by using a large set of simulated observations of the Pan-STARRS project.
\end{abstract}

\section{Introduction}

With the new observational techniques of the next generation surveys, like PanSTARRS and LSST, ${ }^{1}$ the number of moving objects detected in each night of observations is expected to increase by two orders of magnitude with respect to the current surveys. To deal with this huge amount of data the interest in the study of orbit determination methods has been renewed, both from the theoretical and the computational point of view. The classical methods of preliminary

*Giovanni F. Gronchi: Dipartimento di Matematica, Università di Pisa, Largo B. Pontecorvo, 5, Pisa, Italy gronchi@dm.unipi.it

†Linda Dimare: Dipartimento di Matematica, Università di Roma 'La Sapienza', P.le Aldo Moro, 2, Roma, Italy dimare@mat.uniroma1.it

¥Andrea Milani: Dipartimento di Matematica, Università di Pisa, Largo B. Pontecorvo, 5, Pisa, Italy milani@dm.unipi.it

${ }^{1}$ see the web pages http://pan-starrs.ifa.hawaii.edu, and http://www.lsst.org 
orbit determination by Laplace [8] and Gauss [4], that have been often revisited in the last two centuries, see [18], [9], [10], are based on the knowledge of at least three observations of a solar system body in three different nights. Both Laplace's and Gauss' method may produce more than one preliminary orbit for the same object: a detailed analysis of the occurrence of multiple solutions is in [6]. The determination of a preliminary orbit is followed by the differential corrections [1], an iterative method to obtain the minimum of a target function, that improves the orbit in the sense of the least squares fit of the residuals: this sequence of operations was already proposed in [4].

The data of the current surveys generally do not provide a single observation for an object in an observing night: in fact the moving objects are distinguished from fixed stars by detecting them a few times in the same night. The sequence of observations usually gives a too short arc on the celestial sphere [14], such that the data are not enough to compute an orbit for that body. As the number of detected objects per night is very large, it is difficult to decide whether two sequences of observations made in different nights belong to the same object: this gives rise to the problem of linkage of two short arcs of observations.

The information contained in a short arc of observations can be used to define an attributable [11], consisting of the angular position and velocity of the body on the celestial sphere at a given time; the topocentric distance and the radial velocity at that time are unknown. Therefore two short arcs of observations belonging to the same object provide us with 8 scalar data, from which we can try to compute an orbit.

In 1977 Taff and Hall proposed to use the angular momentum and the energy integrals to perform orbit determination starting from a data set that corresponds to two attributables of the same observed body (see [19], [20]). They noticed that the problem can be written in an algebraic form but, since the total degree is high, they suggested to use a Newton-Raphson method to solve the problem. This approach deals with the solutions only locally, and there are alternative possible solutions that can be lost. In this paper we shall start from the same first integrals of the Kepler problem, but we shall exploit the algebraic character of the problem, keeping in this way a global control on the solutions. In particular we shall present two different methods to solve by elimination the polynomial system corresponding to this problem, and to compute all the related preliminary orbits defined by the two attributables.

Since an orbit is defined by 6 scalar data, the available information is redundant, and we can use this to set compatibility conditions for the solutions (see (16)), that should be fulfilled if the two attributables belong to the same solar system object. The unavoidable errors in the observations affect also the computation of the attributables. Given a covariance matrix for the two attributables, expressing their uncertainty, we can use this to compute the value of an identification norm, based on the compatibility conditions, to decide if the attributables may be related to the same body (i.e. if the linkage is successful) and to choose among possible alternative solutions.

The plan of the paper is the following: after introducing some notation related to attributables in Section 2, we explain in Section 3 the orbit determi- 
nation method and derive the bivariate polynomial system whose roots give us the topocentric distances of the observed object at the two epochs. In Section 4 we explain the two algorithms that we propose to search for these roots and the effective computation of the orbits. In Section 5 we deal with the uncertainty of the data, introduce the identification norm and provide the covariance matrices of the preliminary orbits. Some numerical experiments are presented in the last two sections: in Section 6 we show the results of a test case, illustrating the computation of the preliminary orbits for a numbered asteroid whose orbit is well known, while in Section 7 we investigate the performance of the method for a large database of simulated observations.

\section{Attributables}

Let $(\rho, \alpha, \delta) \in \mathbb{R}^{+} \times[-\pi, \pi) \times(-\pi / 2, \pi / 2)$ be spherical coordinates for the topocentric position of a solar system body. The angular coordinates $(\alpha, \delta)$ are defined by a topocentric coordinate system that can be arbitrarily selected. Usually, in the applications, $\alpha$ is the right ascension and $\delta$ the declination with respect to an equatorial coordinate system (e.g., J2000).

Given a short arc of observations of a celestial body $\left(t_{i}, \alpha_{i}, \delta_{i}\right)$, for $i=1 \ldots m$ with $m \geq 2$, it is often possible to compute an attributable, ${ }^{2}$ that is a vector

$$
\mathcal{A}=(\alpha, \delta, \dot{\alpha}, \dot{\delta}) \in[-\pi, \pi) \times(-\pi / 2, \pi / 2) \times \mathbb{R}^{2},
$$

representing the angular position and velocity of the body at a mean time $\bar{t}$ in the selected coordinates (see [11]). Usually we choose $\bar{t}$ as the mean $\left(\sum_{i} t_{i}\right) / m$. The attributable is computed by a polynomial fit, typically linear or quadratic, and the observations used in the computation need to be made by the same observatory. If the observations are enough, i.e. $m \geq 2$ for a linear fit, $m \geq 3$ for a quadratic one, then we can compute also a covariance matrix $\Gamma_{\mathcal{A}}$, representing the uncertainty of the attributable. Note that the topocentric distances $\rho_{i}$ at times $t_{i}$ are completely unknown.

We introduce the heliocentric position and velocity of the body at time $\bar{t}$

$$
\mathbf{r}=\mathbf{q}+\rho \hat{\boldsymbol{\rho}}, \quad \dot{\mathbf{r}}=\dot{\mathbf{q}}+\dot{\rho} \hat{\boldsymbol{\rho}}+\rho\left(\hat{\boldsymbol{\rho}}_{\alpha} \dot{\alpha}+\hat{\boldsymbol{\rho}}_{\delta} \dot{\delta}\right),
$$

with $\rho, \dot{\rho}$ the topocentric distance and the radial velocity, and with $\hat{\boldsymbol{\rho}}, \hat{\boldsymbol{\rho}}_{\alpha}, \hat{\boldsymbol{\rho}}_{\delta}$ the observation direction and its partial derivatives with respect to $\alpha$ and $\delta$.

The vectors $\mathbf{q}, \dot{\mathbf{q}}$ represent the heliocentric position and velocity of the observer on the Earth. The observer position is know as a function of time, but for consistency, if the attributable is computed by a fit to polynomials with low degree, the values $\mathbf{q}(\bar{t}), \dot{\mathbf{q}}(\bar{t})$ need to be computed by the same interpolation. Therefore we make a quadratic fit with the actual geocentric positions $\mathbf{q}\left(t_{i}\right)-\mathbf{q}_{\oplus}\left(t_{i}\right)$ at the times of the individual observations $\left(\mathbf{q}_{\oplus}\right.$ is the heliocentric position of the Earth center) to obtain the interpolating function $\mathbf{q}_{\text {obs }}(t)$; then

\footnotetext{
${ }^{2}$ The name refers to the possibility of attributing the observations of the short arc to an already known orbit.
} 
we take $\mathbf{q}(\bar{t})=\mathbf{q}_{\oplus}(\bar{t})+\mathbf{q}_{\text {obs }}(\bar{t})$ and $\dot{\mathbf{q}}(\bar{t})=\dot{\mathbf{q}}_{\oplus}(\bar{t})+\dot{\mathbf{q}}_{\text {obs }}(\bar{t})$. This method was suggested by Poincaré [18], and it is important to obtain preliminary orbits of better quality, see [15].

In rectangular coordinates we have

$$
\begin{aligned}
& \hat{\boldsymbol{\rho}}=(\cos \alpha \cos \delta, \sin \alpha \cos \delta, \sin \delta), \\
& \hat{\boldsymbol{\rho}}_{\alpha}=(-\sin \alpha \cos \delta, \cos \alpha \cos \delta, 0), \\
& \hat{\boldsymbol{\rho}}_{\delta}=(-\cos \alpha \sin \delta,-\sin \alpha \sin \delta, \cos \delta) .
\end{aligned}
$$

These vectors form an orthogonal system, in particular

$$
|\hat{\boldsymbol{\rho}}|=\left|\hat{\boldsymbol{\rho}}_{\delta}\right|=1, \quad\left|\hat{\boldsymbol{\rho}}_{\alpha}\right|=\cos \delta, \quad \hat{\boldsymbol{\rho}} \cdot \hat{\boldsymbol{\rho}}_{\alpha}=\hat{\boldsymbol{\rho}} \cdot \hat{\boldsymbol{\rho}}_{\delta}=\hat{\boldsymbol{\rho}}_{\alpha} \cdot \hat{\boldsymbol{\rho}}_{\delta}=0,
$$

where the dot indicates the Euclidean scalar product and $|\cdot|$ the corresponding norm.

For later reference we introduce the orthonormal basis $\{\hat{\boldsymbol{\rho}}, \hat{\mathbf{v}}, \hat{\mathbf{n}}\}$ adapted to the apparent path $\hat{\boldsymbol{\rho}}=\hat{\boldsymbol{\rho}}(t)$ of the observed body on the celestial sphere: we define the unit vector $\hat{\mathbf{v}}$ by the relation

$$
\frac{d}{d t} \hat{\boldsymbol{\rho}}=\eta \hat{\mathbf{v}}
$$

where $\eta=\sqrt{\dot{\alpha}^{2} \cos ^{2} \delta+\dot{\delta}^{2}}$ and is called proper motion. Moreover we set $\hat{\mathbf{n}}=$ $\hat{\boldsymbol{\rho}} \times \hat{\mathbf{v}}$.

\section{Linkage by the two-body integrals}

Given two attributables $\mathcal{A}_{1}, \mathcal{A}_{2}$ at different epochs $\bar{t}_{1}, \bar{t}_{2}$, in the hypothesis that they belong to the same observed body, we write down polynomial equations for the topocentric distance and radial velocity of the body at the two epochs by using the angular momentum and the energy integrals.

\subsection{Angular momentum and Energy}

For a given attributable $\mathcal{A}$ the angular momentum vector (per unit mass) can be written as a polynomial function of the radial distance and velocity $\rho, \dot{\rho}$ :

$$
\mathbf{c}(\rho, \dot{\rho})=\mathbf{r} \times \dot{\mathbf{r}}=\mathbf{D} \dot{\rho}+\mathbf{E} \rho^{2}+\mathbf{F} \rho+\mathbf{G},
$$

where

$$
\begin{aligned}
& \mathbf{D}=\mathbf{q} \times \hat{\boldsymbol{\rho}} \\
& \mathbf{E}=\dot{\alpha} \hat{\boldsymbol{\rho}} \times \hat{\boldsymbol{\rho}}_{\alpha}+\dot{\delta} \hat{\boldsymbol{\rho}} \times \hat{\boldsymbol{\rho}}_{\delta}=\eta \hat{\mathbf{n}}, \\
& \mathbf{F}=\dot{\alpha} \mathbf{q} \times \hat{\boldsymbol{\rho}}_{\alpha}+\dot{\delta} \mathbf{q} \times \hat{\boldsymbol{\rho}}_{\delta}+\hat{\boldsymbol{\rho}} \times \dot{\mathbf{q}} \\
& \mathbf{G}=\mathbf{q} \times \dot{\mathbf{q}}
\end{aligned}
$$

depend only on the attributable $\mathcal{A}$ and on the motion of the observer $\mathbf{q}, \dot{\mathbf{q}}$ at the time $\bar{t}$ of the attributable. For the given $\mathcal{A}$ we can also write the two-body energy as a function of $\rho, \dot{\rho}$, as in [12]

$$
2 \mathcal{E}(\rho, \dot{\rho})=\dot{\rho}^{2}+c_{1} \dot{\rho}+c_{2} \rho^{2}+c_{3} \rho+c_{4}-\frac{2 k^{2}}{\sqrt{\rho^{2}+c_{5} \rho+c_{0}}},
$$


where $k$ is Gauss' constant and

$$
\begin{aligned}
& c_{0}=|\mathbf{q}|^{2}, \\
& c_{1}=2 \dot{\mathbf{q}} \cdot \hat{\boldsymbol{\rho}}, \quad c_{2}=\eta^{2}, \\
& c_{3}=2\left(\dot{\alpha} \dot{\mathbf{q}} \cdot \hat{\boldsymbol{\rho}}_{\alpha}+\dot{\delta} \dot{\mathbf{q}} \cdot \hat{\boldsymbol{\rho}}_{\delta}\right), \\
& c_{4}=|\dot{\mathbf{q}}|^{2} \text {, } \\
& c_{5}=2 \mathbf{q} \cdot \hat{\boldsymbol{\rho}},
\end{aligned}
$$

depend only on $\mathcal{A}, \mathbf{q}, \dot{\mathbf{q}}$.

\subsection{Equating the integrals}

Now we take two attributables $\mathcal{A}_{1}=\left(\alpha_{1}, \delta_{1}, \dot{\alpha}_{1}, \dot{\delta}_{1}\right), \mathcal{A}_{2}=\left(\alpha_{2}, \delta_{2}, \dot{\alpha}_{2}, \dot{\delta}_{2}\right)$ at epochs $\bar{t}_{1}, \bar{t}_{2}$; we shall use the notation of Section 3.1, with index 1 or 2 referring to the epoch. If $\mathcal{A}_{1}, \mathcal{A}_{2}$ correspond to the same physical object, then the angular momentum vectors at the two epochs must coincide:

$$
\mathbf{D}_{1} \dot{\rho}_{1}-\mathbf{D}_{2} \dot{\rho}_{2}=\mathbf{J}\left(\rho_{1}, \rho_{2}\right),
$$

where

$$
\mathbf{J}\left(\rho_{1}, \rho_{2}\right)=\mathbf{E}_{2} \rho_{2}^{2}-\mathbf{E}_{1} \rho_{1}^{2}+\mathbf{F}_{2} \rho_{2}-\mathbf{F}_{1} \rho_{1}+\mathbf{G}_{2}-\mathbf{G}_{1} .
$$

Relation (2) is a system of three equations in the four unknowns $\rho_{1}, \dot{\rho}_{1}, \rho_{2}, \dot{\rho}_{2}$, with constraints

$$
\rho_{1}>0, \rho_{2}>0 .
$$

By scalar multiplication of (2) with $\mathbf{D}_{1} \times \mathbf{D}_{2}$ we eliminate the variables $\dot{\rho}_{1}, \dot{\rho}_{2}$ and obtain the equation

$$
\mathbf{D}_{1} \times \mathbf{D}_{2} \cdot \mathbf{J}\left(\rho_{1}, \rho_{2}\right)=0 .
$$

The left hand side in (3) is a quadratic form in the variables $\rho_{1}, \rho_{2}$; we write it as

$$
q\left(\rho_{1}, \rho_{2}\right) \stackrel{\text { def }}{=} q_{20} \rho_{1}^{2}+q_{10} \rho_{1}+q_{02} \rho_{2}^{2}+q_{01} \rho_{2}+q_{00},
$$

with

$$
\begin{array}{rr}
q_{20}=-\mathbf{E}_{1} \cdot \mathbf{D}_{1} \times \mathbf{D}_{2}, & q_{02}=\mathbf{E}_{2} \cdot \mathbf{D}_{1} \times \mathbf{D}_{2}, \\
q_{10}=-\mathbf{F}_{1} \cdot \mathbf{D}_{1} \times \mathbf{D}_{2}, & q_{01}=\mathbf{F}_{2} \cdot \mathbf{D}_{1} \times \mathbf{D}_{2}, \\
q_{00}=\left(\mathbf{G}_{2}-\mathbf{G}_{1}\right) \cdot \mathbf{D}_{1} \times \mathbf{D}_{2} .
\end{array}
$$

Equation (4) defines a conic section in the $\left(\rho_{1}, \rho_{2}\right)$ plane, with symmetry axes parallel to the coordinate axes. Since the directions of $\mathbf{E}_{1}, \mathbf{E}_{2}$ correspond to $\hat{\mathbf{n}}_{1}, \hat{\mathbf{n}}_{2}$, for $\left|\bar{t}_{2}-\bar{t}_{1}\right|$ small enough the angle between these two directions is small and the coefficients $q_{20}, q_{02}$ have opposite signs, thus in this case (4) defines a hyperbola.

We can compute the radial velocities $\dot{\rho}_{1}, \dot{\rho}_{2}$ by vector multiplication of (2) with $\mathbf{D}_{1}$ and $\mathbf{D}_{2}$, projecting on the direction of $\mathbf{D}_{1} \times \mathbf{D}_{2}$ :

$$
\dot{\rho}_{1}\left(\rho_{1}, \rho_{2}\right)=\frac{\left(\mathbf{J} \times \mathbf{D}_{2}\right) \cdot\left(\mathbf{D}_{1} \times \mathbf{D}_{2}\right)}{\left|\mathbf{D}_{1} \times \mathbf{D}_{2}\right|^{2}}, \quad \dot{\rho}_{2}\left(\rho_{1}, \rho_{2}\right)=\frac{\left(\mathbf{J} \times \mathbf{D}_{1}\right) \cdot\left(\mathbf{D}_{1} \times \mathbf{D}_{2}\right)}{\left|\mathbf{D}_{1} \times \mathbf{D}_{2}\right|^{2}} .
$$


For the given $\mathcal{A}_{1}, \mathcal{A}_{2}$ we can also equate the corresponding two-body energies $\mathcal{E}_{1}, \mathcal{E}_{2}$. We use the expressions of $\dot{\rho}_{1}\left(\rho_{1}, \rho_{2}\right), \dot{\rho}_{2}\left(\rho_{1}, \rho_{2}\right)$ above and substitute them into $\mathcal{E}_{1}=\mathcal{E}_{2}$, thus we obtain

$$
\mathcal{F}_{1}\left(\rho_{1}, \rho_{2}\right)-\frac{2 k^{2}}{\sqrt{\mathcal{G}_{1}\left(\rho_{1}\right)}}=\mathcal{F}_{2}\left(\rho_{1}, \rho_{2}\right)-\frac{2 k^{2}}{\sqrt{\mathcal{G}_{2}\left(\rho_{2}\right)}},
$$

for some polynomial functions $\mathcal{F}_{1}\left(\rho_{1}, \rho_{2}\right), \mathcal{F}_{2}\left(\rho_{1}, \rho_{2}\right), \mathcal{G}_{1}\left(\rho_{1}\right), \mathcal{G}_{2}\left(\rho_{2}\right)$ with degrees $\operatorname{deg}\left(\mathcal{F}_{1}\right)=\operatorname{deg}\left(\mathcal{F}_{2}\right)=4$ and $\operatorname{deg}\left(\mathcal{G}_{1}\right)=\operatorname{deg}\left(\mathcal{G}_{2}\right)=2$. By squaring we have

$$
\left(\mathcal{F}_{1}-\mathcal{F}_{2}\right)^{2} \mathcal{G}_{1} \mathcal{G}_{2}-4 k^{4}\left(\mathcal{G}_{1}+\mathcal{G}_{2}\right)=-8 k^{4} \sqrt{\mathcal{G}_{1} \mathcal{G}_{2}} .
$$

Squaring again we obtain the polynomial equation

$$
p\left(\rho_{1}, \rho_{2}\right) \stackrel{\text { def }}{=}\left[\left(\mathcal{F}_{1}-\mathcal{F}_{2}\right)^{2} \mathcal{G}_{1} \mathcal{G}_{2}-4 k^{4}\left(\mathcal{G}_{1}+\mathcal{G}_{2}\right)\right]^{2}-64 k^{8} \mathcal{G}_{1} \mathcal{G}_{2}=0,
$$

with total degree 24. Some spurious solutions may have been added as a result of squaring expressions with unknown sign.

Note that, if the observations were made from the center of the Earth, $\mathbf{G}_{i}(i=1,2)$ would be the angular momentum of the Earth at epochs $\bar{t}_{1}, \bar{t}_{2}$, thus $\mathbf{G}_{1}=\mathbf{G}_{2}$ and $q_{00}=0$. With this simplifying assumption $\rho_{1}=\rho_{2}=0$ is a solution of the system $q\left(\rho_{1}, \rho_{2}\right)=p\left(\rho_{1}, \rho_{2}\right)=0$ that corresponds to the Earth center and therefore is not acceptable. This solution also appears in the geocentric version of the method of Laplace for a preliminary orbit from 3 observations. Actually we use topocentric observations, for which the zero solution is replaced by one with both $\rho_{1}$ and $\rho_{2}$ very small.

\subsection{Degenerate cases}

The quadratic form (4) degenerates into a linear function when

$$
\mathbf{E}_{1} \cdot \mathbf{D}_{1} \times \mathbf{D}_{2}=\mathbf{E}_{2} \cdot \mathbf{D}_{1} \times \mathbf{D}_{2}=0 .
$$

A simple computation shows that

$$
\begin{aligned}
& \mathbf{E}_{1} \cdot \mathbf{D}_{1} \times \mathbf{D}_{2}=\eta_{1}\left(\hat{\mathbf{n}}_{1} \cdot \mathbf{q}_{1}\right)\left(\hat{\boldsymbol{\rho}}_{1} \times \hat{\boldsymbol{\rho}}_{2} \cdot \mathbf{q}_{2}\right), \\
& \mathbf{E}_{2} \cdot \mathbf{D}_{1} \times \mathbf{D}_{2}=\eta_{2}\left(\hat{\mathbf{n}}_{2} \cdot \mathbf{q}_{2}\right)\left(\hat{\boldsymbol{\rho}}_{1} \times \hat{\boldsymbol{\rho}}_{2} \cdot \mathbf{q}_{1}\right),
\end{aligned}
$$

thus, assuming that the proper motions $\eta_{1}, \eta_{2}$ do not vanish and setting $\mathbf{n}_{\mathbf{1 2}}=$ $\hat{\boldsymbol{\rho}}_{1} \times \hat{\boldsymbol{\rho}}_{2}$, the degeneration occurs when either $\mathbf{n}_{\mathbf{1 2}}$ vanishes $(\mathrm{C} 0)$ or at least one the following relations holds:

$$
\begin{aligned}
& \hat{\mathbf{n}}_{1} \cdot \mathbf{q}_{1}=\hat{\mathbf{n}}_{2} \cdot \mathbf{q}_{2}=0, \\
& \mathbf{n}_{\mathbf{1 2}} \cdot \mathbf{q}_{1}=\mathbf{n}_{\mathbf{1 2}} \cdot \mathbf{q}_{2}=0, \\
& \hat{\mathbf{n}}_{1} \cdot \mathbf{q}_{1}=\mathbf{n}_{\mathbf{1 2}} \cdot \mathbf{q}_{1}=0, \\
& \hat{\mathbf{n}}_{2} \cdot \mathbf{q}_{2}=\mathbf{n}_{\mathbf{1 2}} \cdot \mathbf{q}_{2}=0 .
\end{aligned}
$$

The interpretation of these conditions is the following: $(\mathrm{C} 0)$ means that $\hat{\boldsymbol{\rho}}_{1}, \hat{\boldsymbol{\rho}}_{2}$ point to either exactly the same or exactly the opposite direction in the sky; $(\mathrm{C} 1)$ 
means that both sets $\left\{\hat{\boldsymbol{\rho}}_{1}, \hat{\mathbf{v}}_{1}, \mathbf{q}_{1}\right\}$ and $\left\{\hat{\boldsymbol{\rho}}_{2}, \hat{\mathbf{v}}_{2}, \mathbf{q}_{2}\right\}$ are constituted of coplanar vectors; (C2) says that $\hat{\boldsymbol{\rho}}_{1}, \hat{\boldsymbol{\rho}}_{2}, \mathbf{q}_{1}, \mathbf{q}_{2}$ are coplanar. Let us discuss condition (C3): $\hat{\mathbf{n}}_{1} \cdot \mathbf{q}_{1}=0$ means that $\mathbf{q}_{1}, \hat{\boldsymbol{\rho}}_{1}, \hat{\mathbf{v}}_{1}$ are coplanar and $\mathbf{n}_{\mathbf{1 2}} \cdot \mathbf{q}_{1}=0$ means that $\hat{\boldsymbol{\rho}}_{1}, \hat{\boldsymbol{\rho}}_{2}, \mathbf{q}_{1}$ are coplanar as well. If $\mathbf{D}_{1} \neq 0$ we obtain that the four vectors $\mathbf{q}_{1}, \hat{\boldsymbol{\rho}}_{1}, \hat{\mathbf{v}}_{1}, \hat{\boldsymbol{\rho}}_{2}$ all lie in the same plane. In particular (C3) implies that $\hat{\boldsymbol{\rho}}_{2}$ belongs to the great circle defined by the intersection of the plane generated by $\hat{\boldsymbol{\rho}}_{1}, \hat{\mathbf{v}}_{1}$ with the celestial sphere. This degeneration condition can be compared with the failure condition of the classical orbit determination methods with three observations by Gauss and Laplace [17], due to vanishing of the curvature in the apparent path of the observed body on the celestial sphere. The discussion of condition (C4) is similar to the previous one and corresponds to the coplanarity of $\mathbf{q}_{2}, \hat{\boldsymbol{\rho}}_{2}, \hat{\mathbf{v}}_{2}, \hat{\boldsymbol{\rho}}_{1}$.

\section{Computation of the solutions}

In this section we introduce two different methods to search for the solutions of the semi-algebraic problem

$$
\left\{\begin{array}{rl}
p\left(\rho_{1}, \rho_{2}\right) & =0 \\
q\left(\rho_{1}, \rho_{2}\right) & =0
\end{array}, \quad \rho_{1}, \rho_{2}>0\right.
$$

for the polynomials $p, q$ introduced in (8), (4) respectively. Moreover we explain the full procedure for the computation of the preliminary orbits and introduce compatibility conditions to decide whether the attributables used to define the problem are related to the same solar system body.

\subsection{Computation of the resultant via DFT}

The first method consists in writing the resultant (see [3]) of $p$ and $q$ with respect to one variable, say $\rho_{1}$. In this way we find a univariate polynomial in the $\rho_{2}$ variable whose real positive roots are the only possible $\rho_{2}$-components of a solution of (9). By grouping the monomials with the same power of $\rho_{1}$ we can write

$$
\begin{gathered}
p\left(\rho_{1}, \rho_{2}\right)=\sum_{j=0}^{20} a_{j}\left(\rho_{2}\right) \rho_{1}^{j}, \quad \text { where } \\
\operatorname{deg}\left(a_{j}\right)=\left\{\begin{array}{ll}
20 & \text { for } j=0 \ldots 4 \\
24-(j+1) & \text { for } j=2 k-1 \\
24-j & \text { for } j=2 k
\end{array} \text { with } k \geq 3\right.
\end{gathered}
$$

and

$$
q\left(\rho_{1}, \rho_{2}\right)=b_{2} \rho_{1}^{2}+b_{1} \rho_{1}+b_{0}\left(\rho_{2}\right)
$$

for some univariate polynomial coefficients $a_{i}, b_{j}$ depending on $\rho_{2}$ (actually $b_{1}, b_{2}$ are constant). We consider the resultant $\operatorname{Res}\left(\rho_{2}\right)$ of $p, q$ with respect to $\rho_{1}$ : it is 
generically a degree 48 polynomial defined as the determinant of the Sylvester matrix

$$
\mathrm{S}\left(\rho_{2}\right)=\left(\begin{array}{ccccccc}
a_{20} & 0 & b_{2} & 0 & \ldots & \ldots & 0 \\
a_{19} & a_{20} & b_{1} & b_{2} & 0 & \ldots & 0 \\
\vdots & \vdots & b_{0} & b_{1} & b_{2} & \ldots & \vdots \\
\vdots & \vdots & 0 & b_{0} & b_{1} & \ldots & \vdots \\
a_{0} & a_{1} & \vdots & \vdots & \vdots & b_{0} & b_{1} \\
0 & a_{0} & 0 & 0 & 0 & 0 & b_{0}
\end{array}\right) .
$$

The positive real roots of $\operatorname{Res}\left(\rho_{2}\right)$ are the only possible values of $\rho_{2}$ for a solution $\left(\rho_{1}, \rho_{2}\right)$ of $(9)$. We could use the resultant method to eliminate the variable $\rho_{2}$ by a different grouping of the terms of $p, q$ :

$$
p\left(\rho_{1}, \rho_{2}\right)=\sum_{j=0}^{20} a_{j}^{\prime}\left(\rho_{1}\right) \rho_{2}^{j}, \quad q\left(\rho_{1}, \rho_{2}\right)=b_{2}^{\prime} \rho_{2}^{2}+b_{1}^{\prime} \rho_{2}+b_{0}^{\prime}\left(\rho_{1}\right),
$$

where the degrees of $a_{j}^{\prime}$ are described by the same rules as for $a_{j}$.

Apart from non-real and non-positive solutions, we shall see that there are additional different reasons to discard some pairs of solutions of (9), thus we expect that the number of acceptable ones is not large. We use a scheme similar to [5] to compute the coefficients of the resultant $\operatorname{Res}\left(\rho_{2}\right)$ :

1) evaluate $a_{i}\left(\rho_{2}\right), b_{j}\left(\rho_{2}\right)$ at the 64 -th roots of unit $\omega_{k}=e^{2 \pi i \frac{k}{64}}, k=0 \ldots 63$, by a DFT (Discrete Fourier Transform) algorithm;

2) compute the determinant of the 64 Sylvester matrices; by relation

$$
\operatorname{det}\left(\left.\mathrm{S}\left(\rho_{2}\right)\right|_{\rho_{2}=\omega_{k}}\right)=\left.\left(\operatorname{det} \mathrm{S}\left(\rho_{2}\right)\right)\right|_{\rho_{2}=\omega_{k}}
$$

we have the values of $\operatorname{Res}\left(\rho_{2}\right)$ at the 64-th roots of unit;

3) apply an IDFT (Inverse Discrete Fourier Transform) algorithm to obtain the coefficients of $\operatorname{Res}\left(\rho_{2}\right)$ from its evaluations.

The use of the DFT and IDFT allows us to interpolate the resultant $\operatorname{Res}\left(\rho_{2}\right)$ in an efficient way. The use of numerical evaluations, e.g. at the roots of unit, avoids the difficulty of writing a very long symbolic expression for the resultant, that could be cumbersome to be managed by a programming language compiler.

The complete set of complex roots of $\operatorname{Res}\left(\rho_{2}\right)$, with an error bound for each of them, are computed using the algorithm described in [2], which is based on simultaneous iterations. Let $\rho_{2}(k), k=1 \ldots n \leq 48$, be the subset of the real and positive roots of $\operatorname{Res}\left(\rho_{2}\right)$. Then for each $k$ we perform the sequence of operations below:

4) solve the equation $q\left(\rho_{1}, \rho_{2}(k)\right)=0$ and compute the two possible values $\rho_{1}(k, 1), \rho_{1}(k, 2)$ for $\rho_{1}$, discarding negative solutions. Then define $\rho_{1}(k)$ equal to either $\rho_{1}(k, 1)$ or $\rho_{1}(k, 2)$, selecting the one that gives the smaller value of $\left|p\left(\rho_{1}, \rho_{2}(k)\right)\right|$; 
5) discard spurious solutions, resulting from the squaring used to reduce the energy equality to the polynomial equation (8). The spurious solutions are the solutions of (9) that do not satisfy either (7) or (6);

6) compute the corresponding values of $\dot{\rho}_{1}(k), \dot{\rho}_{2}(k)$ by $(5)$ and obtain a pair of orbits defined by the sets $\left(\alpha_{i}, \delta_{i}, \dot{\alpha}_{i}, \dot{\delta}_{i}, \rho_{i}, \dot{\rho}_{i}\right)$ of attributable elements, ${ }^{3}$ for $i=1,2$;

7) change from attributable elements to Cartesian heliocentric coordinates by relation $\mathbf{r}_{i}=\rho_{i}(k) \hat{\boldsymbol{\rho}}_{i}+\mathbf{q}_{i}$ for $i=1,2$, and by the corresponding formula for $\dot{\mathbf{r}}_{i}$. Note that the observer position $\mathbf{q}_{i}$ is not the actual $\mathbf{q}\left(t_{i}\right)$, but is obtained by interpolation as proposed by Poincaré (see Section 2 ). Then a standard coordinate change allows us to obtain the related pairs of orbital elements: we shall use Keplerian elements $(a, e, I, \Omega, \omega, \ell)$, where $\ell$ is the mean anomaly ${ }^{4}$. The epochs of the orbits are $\tilde{t}_{1}(k), \tilde{t}_{2}(k)$, corrected by aberration due to the finite velocity of the light $c$ : $\tilde{t}_{i}(k)=\bar{t}_{i}-\rho_{i}(k) / c$ for $i=1,2$.

We have implemented this algorithm in FORTRAN 90 using quadruple precision for part of these computations, in particular the ones related to DFT and IDFT. This feature appeared necessary to obtain reliable results starting from our first numerical experiments.

\subsection{Normal form of the problem}

Another method to compute the solutions of (9) is based on a coordinate change to variables $\left(\xi_{1}, \xi_{2}\right)$, that allows to perform easily the elimination of either $\xi_{1}$ or $\xi_{2}$. Let us set

$$
p\left(\rho_{1}, \rho_{2}\right)=\sum_{i, j=0}^{20} p_{i, j} \rho_{1}^{i} \rho_{2}^{j} .
$$

First we consider the affine transformation to intermediate variables $\left(\zeta_{1}, \zeta_{2}\right)$

$$
\mathcal{T}:\left(\begin{array}{c}
\rho_{1} \\
\rho_{2}
\end{array}\right) \rightarrow\left(\begin{array}{l}
\zeta_{1} \\
\zeta_{2}
\end{array}\right)=\left(\begin{array}{c}
\sigma_{1}^{-1} \rho_{1}-\tau_{1} \\
\sigma_{2}^{-1} \rho_{2}-\tau_{2}
\end{array}\right)
$$

where, to eliminate the linear terms in (4), we set

$$
\sigma_{1} \tau_{1}=-\frac{q_{1,0}}{2 q_{2,0}} \stackrel{\text { def }}{=} \alpha, \quad \sigma_{2} \tau_{2}=-\frac{q_{0,1}}{2 q_{0,2}} \stackrel{\text { def }}{=} \beta
$$

\footnotetext{
${ }^{3}$ The attributable elements are the same as spherical polar coordinates with their time derivatives: the coordinates are just reordered in such a way that the first four elements form the attributable, hence the name.

${ }^{4}$ Any other set of orbital elements in which the first four are defined by the two-body energy and angular momentum can be used, e.g. cometary elements $\left(p_{d}, e, I, \Omega, \omega, t_{p}\right)$ where $p_{d}$ is the perihelion distance and $t_{p}$ the time of perihelion passage: this set would allow to handle also parabolic and hyperbolic orbits.
} 
so that

$q \circ \mathcal{T}^{-1}\left(\zeta_{1}, \zeta_{2}\right)=q_{2,0} \sigma_{1}^{2}\left[\zeta_{1}^{2}+\frac{q_{0,2} \sigma_{2}^{2}}{q_{2,0} \sigma_{1}^{2}} \zeta_{2}^{2}+\frac{\kappa}{q_{2,0} \sigma_{1}^{2}}\right], \quad$ with $\kappa=q_{0,0}-\frac{q_{1,0}^{2}}{4 q_{2,0}}-\frac{q_{0,1}^{2}}{4 q_{0,2}}$.

If we set, for an arbitrary $\sigma_{2} \in \mathbb{R}$,

$$
\sigma_{1}=\gamma \sigma_{2}, \quad \gamma=\sqrt{-\frac{q_{0,2}}{q_{2,0}}}
$$

we obtain

$$
q \circ \mathcal{T}^{-1}\left(\zeta_{1}, \zeta_{2}\right)=q_{2,0} \sigma_{1}^{2}\left[\zeta_{1}^{2}-\zeta_{2}^{2}-2 c_{\star}\right], \quad \text { with } \quad c_{\star}=-\frac{\kappa}{2 q_{2,0} \sigma_{1}^{2}} .
$$

We already observed that, for $\left|\bar{t}_{2}-\bar{t}_{1}\right|$ small enough, $q_{02}$ and $q_{20}$ have opposite signs, hence in this case the variable change $\mathcal{T}$ is real. However, in general, we have to consider $\mathcal{T}$ as a transformation of the complex domain $\mathbb{C}^{2}$. We also have

$$
p \circ \mathcal{T}^{-1}\left(\zeta_{1}, \zeta_{2}\right)=\sum_{i, j=0}^{20} \tilde{p}_{i, j} \zeta_{1}^{i} \zeta_{2}^{j},
$$

where

$$
\tilde{p}_{i, j}=\sigma_{2}^{i+j} \gamma^{i} \sum_{h=i}^{20} \sum_{k=j}^{20} p_{h, k}\left(\begin{array}{c}
h \\
i
\end{array}\right)\left(\begin{array}{c}
k \\
j
\end{array}\right) \alpha^{h-i} \beta^{k-j},
$$

and $\alpha, \beta, \gamma$ depend only on the coefficients of $q\left(\rho_{1}, \rho_{2}\right)$.

Now we apply a rotation of angle $\pi / 4$ to pass to the $\left(\xi_{1}, \xi_{2}\right)$ variables:

$$
\mathcal{R}:\left(\begin{array}{l}
\zeta_{1} \\
\zeta_{2}
\end{array}\right) \rightarrow\left(\begin{array}{l}
\xi_{1} \\
\xi_{2}
\end{array}\right)=\left[\begin{array}{cc}
\cos \left(\frac{\pi}{4}\right) & -\sin \left(\frac{\pi}{4}\right) \\
\sin \left(\frac{\pi}{4}\right) & \cos \left(\frac{\pi}{4}\right)
\end{array}\right]\left(\begin{array}{l}
\zeta_{1} \\
\zeta_{2}
\end{array}\right) .
$$

We have

$$
\begin{aligned}
q \circ \mathcal{T}^{-1} \circ \mathcal{R}^{-1}\left(\xi_{1}, \xi_{2}\right) & =2 q_{2,0} \sigma_{1}^{2}\left[\xi_{1} \xi_{2}-c_{\star}\right] \\
p \circ \mathcal{T}^{-1} \circ \mathcal{R}^{-1}\left(\xi_{1}, \xi_{2}\right) & =\sum_{i, j=0}^{24} p_{i, j}^{\star} \xi_{1}^{i} \xi_{2}^{j},
\end{aligned}
$$

where

$$
p_{i, j}^{\star}= \begin{cases}\bar{p}_{i+j, i}^{\star} & \text { if } i+j \leq 24 \\ 0 & \text { if } i+j>24\end{cases}
$$

and

$$
\begin{aligned}
\bar{p}_{m, n}^{\star} & =\sum_{h+k=m} \tilde{p}_{h, k} \sum_{i+j=n}\left(\begin{array}{c}
h \\
i
\end{array}\right)\left(\begin{array}{c}
k \\
j
\end{array}\right) \frac{(-1)^{j}}{2^{(h+k) / 2}}= \\
& =\sum_{h=0}^{m} \tilde{p}_{h, m-h} \sum_{i=0}^{n}\left(\begin{array}{c}
h \\
i
\end{array}\right)\left(\begin{array}{c}
m-h \\
n-i
\end{array}\right) \frac{(-1)^{n-i}}{2^{m / 2}}= \\
& =\sum_{h=\max \{m-20,0\}}^{\min \{m, 20\}} \tilde{p}_{h, m-h} \sum_{i=0}^{n}\left(\begin{array}{c}
h \\
i
\end{array}\right)\left(\begin{array}{c}
m-h \\
n-i
\end{array}\right) \frac{(-1)^{n-i}}{2^{m / 2}} .
\end{aligned}
$$


The last equality is obtained taking into account that $\tilde{p}_{h, m-h}=0$ for $h>$ 20 or $m-h>20$. Using the relation $\xi_{1} \xi_{2}=c_{\star}$ we can consider in place of $p \circ \mathcal{T}^{-1} \circ \mathcal{R}^{-1}\left(\xi_{1}, \xi_{2}\right)$ the polynomial

$$
\begin{aligned}
p^{\star}\left(\xi_{1}, \xi_{2}\right) & =\sum_{\substack{h, k=0 \\
h>k}}^{24} p_{h, k}^{\star} c_{\star}^{k} \xi_{1}^{h-k}+\sum_{h=0}^{24} p_{h, h}^{\star} c_{\star}^{h}+\sum_{\substack{h, k=0 \\
h<k}}^{24} p_{h, k}^{\star} c_{\star}^{h} \xi_{2}^{k-h}= \\
& =\sum_{j=1}^{24}\left(\sum_{\substack{h, k=0 \\
h-k=j}}^{24} p_{h, k}^{\star} c_{\star}^{h-j}\right) \xi_{1}^{j}+\sum_{j=1}^{24}\left(\sum_{\substack{h, k=0 \\
k-h=j}}^{24} p_{h, k}^{\star} c_{\star}^{k-j}\right) \xi_{2}^{j}+\sum_{h=0}^{24} p_{h, h}^{\star} c_{\star}^{h}= \\
& =A_{24} \xi_{1}^{24}+\ldots+A_{1} \xi_{1}+A_{0}\left(\xi_{2}\right),
\end{aligned}
$$

with

$$
\begin{gathered}
A_{j}=\sum_{\substack{h, k=0 \\
h-k=j}}^{24} p_{h, k}^{\star} c_{\star}^{h-j}=\sum_{h=j}^{24} p_{h, h-j}^{\star} c_{\star}^{h-j}, \quad j=1 \ldots 24, \\
A_{0}\left(\xi_{2}\right)=B_{24} \xi_{2}^{24}+\ldots+B_{1} \xi_{2}+B_{0}, \\
B_{j}=\sum_{\substack{h, k=0 \\
k-h=j}}^{24} p_{h, k}^{\star} c_{\star}^{k-j}=\sum_{k=j}^{24} p_{k-j, k}^{\star} c_{\star}^{k-j}, \quad j=0 \ldots 24 .
\end{gathered}
$$

We consider the algebraic problem in normal form

$$
\left\{\begin{array}{l}
p^{\star}\left(\xi_{1}, \xi_{2}\right)=0 \\
\xi_{1} \xi_{2}-c_{\star}=0
\end{array}\right.
$$

In this case we have to consider all the solutions of (15), not only the ones with real and positive components.

If $c_{\star}=0$, then the solutions $\left(\xi_{1}, \xi_{2}\right)$ of $(15)$ are of the form $\left(\xi_{1}(k), 0\right)$ or $\left(0, \xi_{2}(k)\right)$, where $\xi_{1}(k), \xi_{2}(k), k=1 \ldots 24$ are the roots of $A_{24} \xi_{1}^{24}+\ldots+A_{1} \xi_{1}+B_{0}$ and $B_{24} \xi_{2}^{24}+\ldots+B_{1} \xi_{2}+B_{0}$ respectively.

If $c_{\star} \neq 0$, using the relation $\xi_{1} \xi_{2}=c_{\star}$ we can eliminate one variable, say $\xi_{1}$, from $p^{\star}$. Thus we obtain the univariate polynomial

$$
\mathfrak{p}\left(\xi_{2}\right)=\sum_{k=0}^{48} \mathfrak{p}_{k} \xi_{2}^{k}, \quad \text { with } \mathfrak{p}_{k}= \begin{cases}A_{24-k} c_{\star}^{24-k}, & 0 \leq k \leq 23 \\ B_{k-24}, & 24 \leq k \leq 48\end{cases}
$$

We compute all the complex roots $\xi_{2}(k), k=1 \ldots 48$ of $\mathfrak{p}\left(\xi_{2}\right)$ by the algorithm in [2]; then for each $k$ we define the other component of the solution by

$$
\xi_{1}(k)=\frac{c_{\star}}{\xi_{2}(k)} .
$$

Given all the complex solutions of (15) we compute the corresponding points in the $\left(\rho_{1}, \rho_{2}\right)$ plane by

$$
\left(\rho_{1}(k), \rho_{2}(k)\right)=\mathcal{T}^{-1} \circ \mathcal{R}^{-1}\left(\xi_{1}(k), \xi_{2}(k)\right), \quad k=1 \ldots 48,
$$


discarding the ones with non-real or non-positive components. At this point the preliminary orbits can be computed following the same steps 5),6), 7) of the algorithm explained in Subsection 4.1.

From a few experiments performed this method seems to require more than quadruple precision because of the complicated formulae defining the transformation used to obtain the normal form (15). Thus the advantage in the simple elimination of the variable $\xi_{1}$ must be balanced with the introduction of heavier computations.

\subsection{Compatibility conditions}

The knowledge of the angular momentum vector and of the energy at a given time allows us to compute the Keplerian elements

$$
a, e, I, \Omega \text {. }
$$

In fact the semimajor axis $a$ and the eccentricity $e$ can be computed from the energy and the size of the angular momentum through the relations

$$
\mathcal{E}=-\frac{k^{2}}{2 a}, \quad\|\mathbf{c}\|=k \sqrt{a\left(1-e^{2}\right)} ;
$$

the longitude of the node $\Omega$ and the inclination $I$ are obtained from the direction of the angular momentum

$$
\hat{\mathbf{c}}=(\sin \Omega \sin I,-\cos \Omega \sin I, \cos I) .
$$

The two attributables $\mathcal{A}_{1}, \mathcal{A}_{2}$ at epochs $\bar{t}_{1}, \bar{t}_{2}$ give 8 scalar data, thus the problem is over-determined. From a non-spurious pair $\left(\tilde{\rho}_{1}, \tilde{\rho}_{2}\right)$, solution of $(9)$, we obtain the same values of $a, e, I, \Omega$ at both times $\tilde{t}_{i}, i=1,2$, but we must check that the orbit is indeed the same, that is check the compatibility conditions

$$
\omega_{1}=\omega_{2}, \quad \ell_{1}=\ell_{2}+n\left(\tilde{t}_{1}-\tilde{t}_{2}\right),
$$

where $\omega_{1}, \omega_{2}$ and $\ell_{1}, \ell_{2}$ are the arguments of perihelion and the mean anomalies of the body at times $\tilde{t}_{1}, \tilde{t}_{2}$ and $n=k a^{-3 / 2}$ is the mean motion, which is the same for the two orbits. The first of conditions (16) corresponds to the use of the fifth integral of the Kepler problem, related to Lenz-Laplace's integral vector

$$
\mathbf{L}=\frac{1}{k^{2}} \dot{\mathbf{r}} \times \mathbf{c}-\frac{\mathbf{r}}{|\mathbf{r}|} .
$$

Indeed the compatibility conditions (16) can not be exactly satisfied, due to both the errors in the observations and to the planetary perturbations. Actually the latter are important only when the observed body undergoes a close approach to some planet in the interval between $\tilde{t}_{1}$ and $\tilde{t}_{2}$. Thus we may be able to discard some solutions, for which the compatibility conditions are largely violated. Nevertheless, we need a criterion to assess whether smaller discrepancies from the exact conditions (16) are due to the measurement uncertainty or rather due to the fact that the two attributables do not belong to the same physical object. This will be introduced in the next section. 


\section{Covariance of the solutions}

Given a pair of attributables $\mathbf{A}=\left(\mathcal{A}_{1}, \mathcal{A}_{2}\right)$ with covariance matrices $\Gamma_{\mathcal{A}_{1}}, \Gamma_{\mathcal{A}_{2}}$, we call $\mathbf{R}=\left(\rho_{1}, \dot{\rho}_{1}, \rho_{2}, \dot{\rho}_{2}\right)$ one of the solutions of the equation $\mathbf{\Phi}(\mathbf{R} ; \mathbf{A})=\mathbf{0}$, with

$$
\boldsymbol{\Phi}(\mathbf{R} ; \mathbf{A})=\left(\begin{array}{c}
\mathbf{D}_{1} \dot{\rho}_{1}-\mathbf{D}_{2} \dot{\rho}_{2}-\mathbf{J}\left(\rho_{1}, \rho_{2}\right) \\
\mathcal{E}_{1}\left(\rho_{1}, \dot{\rho}_{1}\right)-\mathcal{E}_{2}\left(\rho_{2}, \dot{\rho}_{2}\right)
\end{array}\right)
$$

We can repeat what follows for each solution of $\mathbf{\Phi}(\mathbf{R} ; \mathbf{A})=\mathbf{0}$.

Let $\mathbf{R}=\mathbf{R}(\mathbf{A})=\left(\mathcal{R}_{1}(\mathbf{A}), \mathcal{R}_{2}(\mathbf{A})\right)$, where $\mathcal{R}_{i}(\mathbf{A})=\left(\rho_{i}(\mathbf{A}), \dot{\rho}_{i}(\mathbf{A})\right)$ for $i=$ 1,2 . If both the elements $\left(\mathcal{A}_{1}, \mathcal{R}_{1}(\mathbf{A})\right),\left(\mathcal{A}_{2}, \mathcal{R}_{2}(\mathbf{A})\right)$ give negative two-body energy orbits, then we can compute the corresponding Keplerian elements at times

$$
\tilde{t}_{i}=\tilde{t}_{i}(\mathbf{A})=\bar{t}_{i}-\frac{\rho_{i}(\mathbf{A})}{c}, \quad i=1,2
$$

through the transformation

$$
(\alpha, \delta, \dot{\alpha}, \dot{\delta}, \rho, \dot{\rho})=(\mathcal{A}, \mathcal{R}) \mapsto \mathcal{E}_{K e p}(\mathcal{A}, \mathcal{R})=(a, e, I, \Omega, \omega, \ell)
$$

We have, for example, a smooth function

$$
\omega_{i}=\omega_{i}(\mathbf{A})=\omega\left(\mathcal{A}_{i}, \mathcal{R}_{i}(\mathbf{A})\right), \quad i=1,2
$$

and similar functional relations for $a, e, I, \Omega, \ell$. Actually, by construction, we have $a_{1}=a_{2}, e_{1}=e_{2}, I_{1}=I_{2}, \Omega_{1}=\Omega_{2}$ : we denote by a the common value of $a_{1}$ and $a_{2}$. We use the vector differences

$$
\Delta_{1,2}=(\Delta \omega, \Delta \ell)
$$

where $\Delta \omega$ is the difference of the two angles $\omega_{1}$ and $\omega_{2}, \Delta \ell$ is the difference of the two angles $\ell_{1}$ and $\ell_{2}+\mathrm{n}\left(\tilde{t}_{1}-\tilde{t}_{2}\right)$ and $\mathrm{n}=k \mathrm{a}^{-3 / 2}$ is the mean motion of both orbits. Here we compute the difference of two angles in such a way that it is a smooth function near a vanishing point; for example we define $\Delta \omega=\left[\omega_{1}-\omega_{2}+\pi(\bmod 2 \pi)\right]-\pi$. With this caution, the vector $\Delta_{1,2}=\Delta_{1,2}(\mathbf{A})$ represents the discrepancy in perihelion argument and mean anomaly of the two orbits, comparing the anomalies at the same time $\tilde{t}_{1}$. We introduce the map

$$
\begin{gathered}
\Psi:\left([-\pi, \pi) \times\left(-\frac{\pi}{2}, \frac{\pi}{2}\right) \times \mathbb{R}^{2}\right)^{2} \longrightarrow[-\pi, \pi) \times\left(-\frac{\pi}{2}, \frac{\pi}{2}\right) \times \mathbb{R}^{2} \times \mathbb{R}^{+} \times \mathbb{R} \times S^{1} \times S^{1} \\
\left(\mathcal{A}_{1}, \mathcal{A}_{2}\right)=\mathbf{A} \mapsto \mathbf{\Psi}(\mathbf{A})=\left(\mathcal{A}_{1}, \mathcal{R}_{1}, \Delta_{1,2}\right)
\end{gathered}
$$

giving the orbit $\left(\mathcal{A}_{1}, \mathcal{R}_{1}(\mathbf{A})\right)$ in attributable elements at time $\tilde{t}_{1}$ (the epoch of the first attributable corrected by aberration), together with the difference $\Delta_{1,2}(\mathbf{A})$ in the angular elements, which are not constrained by the angular momentum and the energy integrals. By the covariance propagation rule we have

$$
\Gamma_{\Psi(\mathbf{A})}=\frac{\partial \boldsymbol{\Psi}}{\partial \mathbf{A}} \Gamma_{\mathbf{A}}\left[\frac{\partial \boldsymbol{\Psi}}{\partial \mathbf{A}}\right]^{T}
$$


where

$$
\frac{\partial \Psi}{\partial \mathbf{A}}=\left[\begin{array}{cc}
I & 0 \\
\frac{\partial \mathcal{R}_{1}}{\partial \mathcal{A}_{1}} & \frac{\partial \mathcal{R}_{1}}{\partial \mathcal{A}_{2}} \\
\frac{\partial \Delta_{1,2}}{\partial \mathcal{A}_{1}} & \frac{\partial \Delta_{1,2}}{\partial \mathcal{A}_{2}}
\end{array}\right] \quad \text { and } \quad \Gamma_{\mathbf{A}}=\left[\begin{array}{cc}
\Gamma_{\mathcal{A}_{1}} & 0 \\
0 & \Gamma_{\mathcal{A}_{2}}
\end{array}\right]
$$

so that the covariance of $\boldsymbol{\Psi}(\mathbf{A})$ is given by the $8 \times 8$ matrix

$$
\Gamma_{\Psi(\mathbf{A})}=\left[\begin{array}{ccc}
\Gamma_{\mathcal{A}_{1}} & \Gamma_{\mathcal{A}_{1}, \mathcal{R}_{1}} & \Gamma_{\mathcal{A}_{1}, \Delta_{1,2}} \\
\Gamma_{\mathcal{R}_{1}, \mathcal{A}_{1}} & \Gamma_{\mathcal{R}_{1}} & \Gamma_{\mathcal{R}_{1}, \Delta_{1,2}} \\
\Gamma_{\Delta_{1,2}, \mathcal{A}_{1}} & \Gamma_{\Delta_{1,2}, \mathcal{R}_{1}} & \Gamma_{\Delta_{1,2}}
\end{array}\right],
$$

where

$$
\begin{gathered}
\Gamma_{\mathcal{A}_{1}, \mathcal{R}_{1}}=\Gamma_{\mathcal{A}_{1}}\left[\frac{\partial \mathcal{R}_{1}}{\partial \mathcal{A}_{1}}\right]^{T}, \quad \Gamma_{\mathcal{A}_{1}, \Delta_{1,2}}=\Gamma_{\mathcal{A}_{1}}\left[\frac{\partial \Delta_{1,2}}{\partial \mathcal{A}_{1}}\right]^{T}, \\
\Gamma_{\mathcal{R}_{1}, \Delta_{1,2}}=\frac{\partial \mathcal{R}_{1}}{\partial \mathcal{A}_{1}} \Gamma_{\mathcal{A}_{1}}\left[\frac{\partial \Delta_{1,2}}{\partial \mathcal{A}_{1}}\right]^{T}+\frac{\partial \mathcal{R}_{1}}{\partial \mathcal{A}_{2}} \Gamma_{\mathcal{A}_{2}}\left[\frac{\partial \Delta_{1,2}}{\partial \mathcal{A}_{2}}\right]^{T}, \\
\Gamma_{\mathcal{R}_{1}, \mathcal{A}_{1}}=\Gamma_{\mathcal{A}_{1}, \mathcal{R}_{1}}^{T}, \quad \Gamma_{\Delta_{1,2}, \mathcal{A}_{1}}=\Gamma_{\mathcal{A}_{1}, \Delta_{1,2}}^{T}, \quad \Gamma_{\Delta_{1,2}, \mathcal{R}_{1}}^{T}=\Gamma_{\mathcal{R}_{1}, \Delta_{1,2}}^{T},
\end{gathered}
$$

and

$\Gamma_{\mathcal{A}_{1}}=\frac{\partial \mathcal{A}_{1}}{\partial \mathbf{A}} \Gamma_{\mathbf{A}}\left[\frac{\partial \mathcal{A}_{1}}{\partial \mathbf{A}}\right]^{T}, \quad \Gamma_{\mathcal{R}_{1}}=\frac{\partial \mathcal{R}_{1}}{\partial \mathbf{A}} \Gamma_{\mathbf{A}}\left[\frac{\partial \mathcal{R}_{1}}{\partial \mathbf{A}}\right]^{T}, \quad \Gamma_{\Delta_{1,2}}=\frac{\partial \Delta_{1,2}}{\partial \mathbf{A}} \Gamma_{\mathbf{A}}\left[\frac{\partial \Delta_{1,2}}{\partial \mathbf{A}}\right]^{T}$.

The matrices $\frac{\partial \mathcal{R}_{i}}{\partial \mathcal{A}_{j}}, i, j=1,2$, can be computed from the relation

$$
\frac{\partial \mathbf{R}}{\partial \mathbf{A}}(\mathbf{A})=-\left[\frac{\partial \Phi}{\partial \mathbf{R}}(\mathbf{R}(\mathbf{A}), \mathbf{A})\right]^{-1} \frac{\partial \Phi}{\partial \mathbf{A}}(\mathbf{R}(\mathbf{A}), \mathbf{A}) .
$$

We also have

$$
\begin{aligned}
& \frac{\partial \Delta \omega}{\partial \mathcal{A}_{1}}=\frac{\partial \omega}{\partial \mathcal{A}}\left(\mathcal{A}_{1}, \mathcal{R}_{1}(\mathbf{A})\right)+\frac{\partial \omega}{\partial \mathcal{R}}\left(\mathcal{A}_{1}, \mathcal{R}_{1}(\mathbf{A})\right) \frac{\partial \mathcal{R}_{1}(\mathbf{A})}{\partial \mathcal{A}_{1}}-\frac{\partial \omega}{\partial \mathcal{R}}\left(\mathcal{A}_{2}, \mathcal{R}_{2}(\mathbf{A})\right) \frac{\partial \mathcal{R}_{2}(\mathbf{A})}{\partial \mathcal{A}_{1}}, \\
& \frac{\partial \Delta \omega}{\partial \mathcal{A}_{2}}=\frac{\partial \omega}{\partial \mathcal{R}}\left(\mathcal{A}_{1}, \mathcal{R}_{1}(\mathbf{A})\right) \frac{\partial \mathcal{R}_{1}(\mathbf{A})}{\partial \mathcal{A}_{2}}-\frac{\partial \omega}{\partial \mathcal{A}}\left(\mathcal{A}_{2}, \mathcal{R}_{2}(\mathbf{A})\right)-\frac{\partial \omega}{\partial \mathcal{R}}\left(\mathcal{A}_{2}, \mathcal{R}_{2}(\mathbf{A})\right) \frac{\partial \mathcal{R}_{2}(\mathbf{A})}{\partial \mathcal{A}_{2}}
\end{aligned}
$$

and

$$
\begin{aligned}
\frac{\partial \Delta \ell}{\partial \mathcal{A}_{1}} & =\frac{\partial \ell}{\partial \mathcal{A}}\left(\mathcal{A}_{1}, \mathcal{R}_{1}(\mathbf{A})\right)+\frac{\partial \ell}{\partial \mathcal{R}}\left(\mathcal{A}_{1}, \mathcal{R}_{1}(\mathbf{A})\right) \frac{\partial \mathcal{R}_{1}(\mathbf{A})}{\partial \mathcal{A}_{1}}-\frac{\partial \ell}{\partial \mathcal{R}}\left(\mathcal{A}_{2}, \mathcal{R}_{2}(\mathbf{A})\right) \frac{\partial \mathcal{R}_{2}(\mathbf{A})}{\partial \mathcal{A}_{1}}+ \\
& +\frac{3}{2} \frac{\mathrm{n}}{\mathrm{a}}\left[\frac{\partial a}{\partial \mathcal{A}}\left(\mathcal{A}_{1}, \mathcal{R}_{1}(\mathbf{A})\right)+\frac{\partial a}{\partial \mathcal{R}}\left(\mathcal{A}_{1}, \mathcal{R}_{1}(\mathbf{A})\right) \frac{\partial \mathcal{R}_{1}(\mathbf{A})}{\partial \mathcal{A}_{1}}\right]\left[\tilde{t}_{1}(\mathbf{A})-\tilde{t}_{2}(\mathbf{A})\right]+ \\
& +\frac{\mathrm{n}}{c}\left[\frac{\partial \rho_{1}}{\partial \mathcal{A}_{1}}(\mathbf{A})-\frac{\partial \rho_{2}}{\partial \mathcal{A}_{1}}(\mathbf{A})\right]
\end{aligned}
$$




$$
\begin{aligned}
\frac{\partial \Delta \ell}{\partial \mathcal{A}_{2}} & =\frac{\partial \ell}{\partial \mathcal{R}}\left(\mathcal{A}_{1}, \mathcal{R}_{1}(\mathbf{A})\right) \frac{\partial \mathcal{R}_{1}(\mathbf{A})}{\partial \mathcal{A}_{2}}-\frac{\partial \ell}{\partial \mathcal{A}}\left(\mathcal{A}_{2}, \mathcal{R}_{2}(\mathbf{A})\right)-\frac{\partial \ell}{\partial \mathcal{R}}\left(\mathcal{A}_{2}, \mathcal{R}_{2}(\mathbf{A})\right) \frac{\partial \mathcal{R}_{2}(\mathbf{A})}{\partial \mathcal{A}_{2}}+ \\
& +\frac{3}{2} \frac{\mathrm{n}}{\mathrm{a}}\left[\frac{\partial a}{\partial \mathcal{A}}\left(\mathcal{A}_{2}, \mathcal{R}_{2}(\mathbf{A})\right)+\frac{\partial a}{\partial \mathcal{R}}\left(\mathcal{A}_{2}, \mathcal{R}_{2}(\mathbf{A})\right) \frac{\partial \mathcal{R}_{2}(\mathbf{A})}{\partial \mathcal{A}_{2}}\right]\left[\tilde{t}_{1}(\mathbf{A})-\tilde{t}_{2}(\mathbf{A})\right]+ \\
& +\frac{\mathrm{n}}{c}\left[\frac{\partial \rho_{1}}{\partial \mathcal{A}_{2}}(\mathbf{A})-\frac{\partial \rho_{2}}{\partial \mathcal{A}_{2}}(\mathbf{A})\right]
\end{aligned}
$$

\subsection{Identification of attributables}

One important step is to decide if trying to link $\mathcal{A}_{1}, \mathcal{A}_{2}$ has produced at least one reliable orbit, so that we can state the two sets of observations defining the $\mathcal{A}_{i}, i=1,2$ may belong to one and the same solar system body. Neglecting the unavoidable errors in the observations and the approximations made both with the interpolation to compute $\mathcal{A}_{1}, \mathcal{A}_{2}$ and with the use of a two-body model, if the observations belong to the same solar system body, then $\Delta_{1,2}(\mathbf{A})=\mathbf{0}$. We need to check whether the failure of this condition is within the acceptable range of values which is statistically expected to be generated by the errors in the available observations.

The marginal covariance matrix of the compatibility conditions is

$$
\Gamma_{\Delta_{1,2}}=\frac{\partial \Delta_{1,2}}{\partial \mathbf{A}} \Gamma_{\mathbf{A}}\left[\frac{\partial \Delta_{1,2}}{\partial \mathbf{A}}\right]^{T}
$$

The inverse matrix $C^{\Delta_{1,2}}=\Gamma_{\Delta_{1,2}}^{-1}$ defines a norm $\|\cdot\|_{\star}$ in the $(\Delta \omega, \Delta \ell)$ plane, allowing to test an identification between the attributables $\mathcal{A}_{1}, \mathcal{A}_{2}$ : the test is

$$
\left\|\Delta_{1,2}\right\|_{\star}^{2}=\Delta_{1,2} C^{\Delta_{1,2}} \Delta_{1,2}^{T} \leq \chi_{\max }^{2},
$$

where $\chi_{\max }$ is a control parameter. The value of the control could be selected on the basis of $\chi^{2}$ tables, if we could assume that the observations errors are Gaussian and their standard deviations, mean values and correlations were known. Since this hypothesis is not satisfied in practice, the control value $\chi_{\text {max }}$ needs to be selected on the basis of large scale tests. Note that, for each pair of attributables, more than one preliminary orbit computed with the method of Section 4 could pass the control (19); thus we can have alternative preliminary orbits.

\subsection{Uncertainty of the orbits}

The methods explained in Section 4 also allow to assign an uncertainty to the preliminary orbits that we compute from the two attributables. A solution $\left(\mathcal{A}_{1}, \mathcal{R}_{1}(\mathbf{A})\right)$, in attributable elements, has the marginal covariance matrix

$$
\left[\begin{array}{cc}
\Gamma_{\mathcal{A}_{1}} & \Gamma_{\mathcal{A}_{1}, \mathcal{R}_{1}} \\
\Gamma_{\mathcal{R}_{1}, \mathcal{A}_{1}} & \Gamma_{\mathcal{R}_{1}}
\end{array}\right]
$$

The preliminary orbits obtained by the other available algorithms do not produce a nondegenerate covariance matrix: this is usually computed in the differential correction step of the orbit determination procedure. With the algorithm 
of [13] a covariance matrix may be defined, but it is not positive definite. The advantage of having a covariance matrix already from the preliminary orbit step could be important in two ways. First, the covariance matrix describes a confidence ellipsoid where a two-body orbit, compatible with the observations and their errors, can be found. The size of this ellipsoid can provide useful hints on the difficulty of the differential corrections procedure. Second, even if the differential corrections are divergent, the covariance matrix of the preliminary orbit can be used to compute a prediction with confidence region, allowing for a planned recovery, for assessment of impact risk, and so on.

\section{A test case}

We show a test of the linkage procedure using the attributables

$$
\begin{aligned}
& \mathcal{A}_{1}=(0.2872656,0.1106342,-0.00375115,-0.00167695) \\
& \mathcal{A}_{2}=(0.2820817,0.1086542,0.00514465,0.00215975)
\end{aligned}
$$

of the asteroid (101878) $1999 \mathrm{NR}_{23}$ at epochs $\bar{t}_{1}=54000, \bar{t}_{2}=54109$ respectively (time in MJD). The values of the components of $\mathcal{A}_{1}, \mathcal{A}_{2}$ are in radians/radians per day. They have been computed from two groups of observations, separated by more than 100 days, made from two different observatories: Mauna Kea (568) and Mt. Lemmon Survey (G96). From the known nominal orbit of this asteroid we obtain the values

$$
\rho_{1}=1.0419, \quad \rho_{2}=2.0485
$$

of the topocentric distance (in AU) at the two mean epochs of the observations.

In Figure 1 we show the intersections between the curves defined by $p\left(\rho_{1}, \rho_{2}\right)$ and $q\left(\rho_{1}, \rho_{2}\right)$. By solving the corresponding problem (9) with the method described in Subsection 4.1 we find the 6 positive pairs of solutions $\left(\rho_{1}, \rho_{2}\right)$ displayed in Table 1. After removing solution 1 (with both components very small),

\begin{tabular}{c|c|c} 
& $\rho_{1}$ & $\rho_{2}$ \\
\hline 1 & 0.0059 & 0.0097 \\
2 & 0.7130 & 1.4100 \\
3 & 0.7045 & 1.3933 \\
4 & 1.0409 & 2.0517 \\
5 & 1.1659 & 2.2952 \\
6 & 1.4246 & 2.7968
\end{tabular}

Table 1: Solutions of the system (9) for (101878) $1999 \mathrm{NR}_{23}$.

the spurious solution 6 (not satisfying (7)) and the spurious solutions 3 and 5 (not satisfying (6)), we are left with the values labeled 2 and 4 in Table 1 . Note that, even if solutions 2 and 3 look close, they are far apart enough to select 


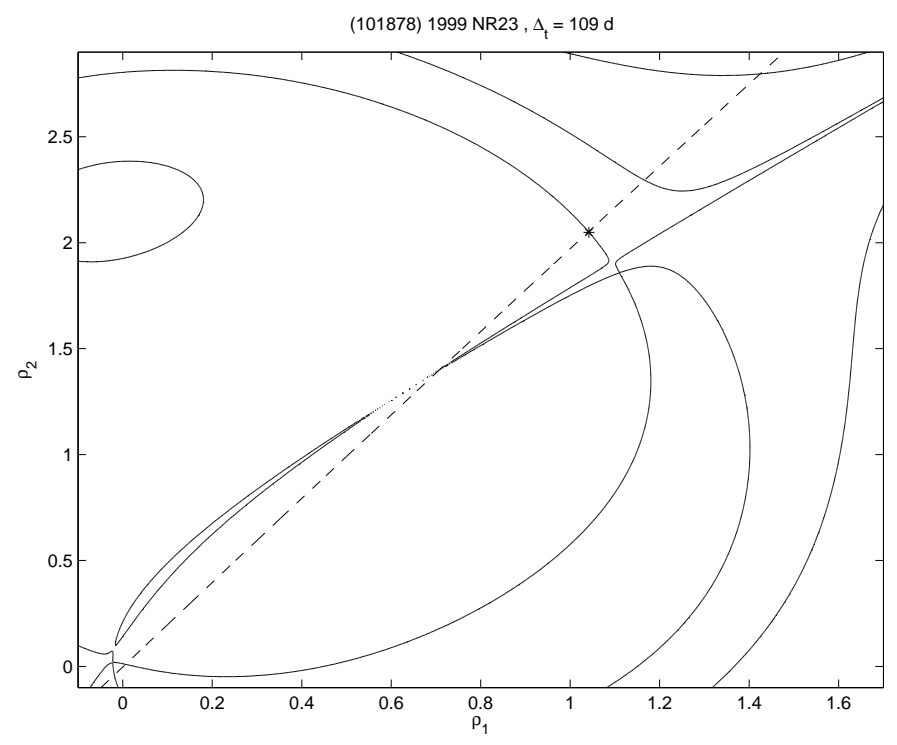

Figure 1: Intersections of the curves $p=0, q=0$ (solid and dashed, respectively) in the plane $\rho_{1}, \rho_{2}$ for the asteroid (101878) $1999 \mathrm{NR}_{23}$ : the asterisk corresponds to the true orbit.

\begin{tabular}{c|r|l|r|l|} 
& \multicolumn{2}{|c|}{1} & \multicolumn{2}{c|}{2} \\
\hline$\left\|\Delta_{1,2}\right\|_{\star}$ & \multicolumn{2}{|c|}{487.65806} & 2.25828 & 2.25828 \\
\hline$a$ & 6.87384 & 6.87384 & 0.19787 & 0.19787 \\
$e$ & 0.81798 & 0.81798 & 0.59995 & 0.59995 \\
$I$ & 0.51733 & 0.51733 & 156.42531 & 156.42531 \\
$\Omega$ & 156.55521 & 156.55521 & 144.39580 & 145.26330 \\
$\omega$ & 144.68146 & 321.78289 & 47.75173 & 78.65378 \\
$\ell$ & 4.66178 & 355.27766 & 53999.8186 & 54109.1331
\end{tabular}

Table 2: Keplerian elements (angles in degrees) corresponding to the pairs $\left(\rho_{1}, \rho_{2}\right)$ labeled with 2 and 4 in Table 1 . The value of $\left\|\Delta_{1,2}\right\|_{\star}$ is shown for the two solutions.

only one of them as a good solution. For the remaining solutions 2 and 4 we succeed in computing Keplerian orbits, that we list in Table 2. The values of $a, e, I, \Omega$ are the same for each pair: this is due to the fact that each pair of orbits shares the same angular momentum and the same energy. The value of the identification norm $\left\|\Delta_{1,2}\right\|_{\star}$, also shown in Table 2 , strongly suggests to select the second solution. The results appear pretty good, in fact the differences with the true solution are of the order of $3 \times 10^{-5}$ AU and the errors in the Keplerian elements are comparable with the planetary perturbations; this is intrinsically 
bound to the use of a two-body approximation.

\section{Numerical experiments with simulated obser- vations}

We have tested our identification method with the DFT algorithm, explained in Subsection 4.1, using simulated observations of objects in a solar system model. The data have been given to us by R. Jedicke and L. Dennau from the Institute of Astronomy, University of Hawaii, and the data quality resemble the one which should be achieved by the Pan-STARRS telescope when it will be fully operative. The RMS of the observations vary from 0.01 to 0.02 arcsec, that is rather optimistic for the current surveys. The current astrometric data quality of the Pan-STARRS 1 telescope is such that the RMS of the residuals for well determined asteroid orbits is between 0.11 and 0.13 arcsec. Better results should be achieved when the astrometric reduction of asteroid detections will be performed with respect to a catalogue generated by the Pan-STARRS survey itself.

These observations cover 31 observing nights, in three consecutive lunations and are grouped into tracklets. Each tracklet is composed by observations presumably belonging to the same object and covering a short arc: some of them are false (e.g. join observations of different objects). From each tracklet we can compute an attributable. We have first applied to the database of tracklets the identification procedures defined in [13], [7]. Then we have tested our method on the leftover database, for which the previous procedure has failed. These remaining observations corresponds to 19441 objects, and 24590 tracklets, but only 4132 objects have at least two tracklets, that is a necessary requirement for the application of our method. The hyperbolic orbits have been removed from the solar system model: in fact our current method does not search for them, but it could be easily modified to include their orbit determination. To each accepted preliminary orbit obtained from a pair of attributables we apply the differential corrections, using all the observations at our disposal, to compute a least squares orbit with its covariance matrix. ${ }^{5}$

To reduce the computational complexity, we need to define a filter selecting the pairs of attributables which we try to link. In Section 7.1 we describe the two filters we have used in processing the simulated data.

\subsection{Filtering pairs of attributables}

\subsubsection{First filter: guessing the second angular position.}

A first simple way to discard pairs of attributables at epochs $\bar{t}_{1}, \bar{t}_{2}$ is to constrain the time span $\delta t=\bar{t}_{2}-\bar{t}_{1}$ : we require

$$
\delta t_{\min } \leq \delta t \leq \delta t_{\max }
$$

${ }^{5}$ We use the preliminary orbit at time $\tilde{t}_{1}$ as starting guess for the differential corrections. We could also use the orbit at time $\tilde{t}_{2}$, or an 'average orbit' at time $\left(\tilde{t}_{1}+\tilde{t}_{2}\right) / 2$. 
for suitable positive constants $\delta t_{\min }, \delta t_{\max }$. In our experiment we have used $\delta t_{\text {max }}=99$ days and $\delta t_{\text {min }}=0.5$ days, that practically means we have tried to link attributables obtained in different nights. For each given pair of attributables at epochs $\bar{t}_{1}, \bar{t}_{2}$ fulfilling (20) we consider for $i=1,2$ the corresponding proper motions $\eta_{i}$ and the mobile bases $\left\{\hat{\boldsymbol{\rho}}_{i}, \hat{\mathbf{v}}_{i}, \hat{\mathbf{n}}_{i}\right\}$, defined in Section 2. We want to use one of the proper motions, say $\eta_{1}$, to bound the region in the sky where we could recover the object at the other time $\bar{t}_{2}$.

Let us form the orthogonal matrices $V_{1}=\left[\hat{\boldsymbol{\rho}}_{1}\left|\hat{\mathbf{v}}_{1}\right| \hat{\mathbf{n}}_{1}\right]$ and $V_{2}=\left[\hat{\boldsymbol{\rho}}_{2}\left|\hat{\mathbf{v}}_{2}\right| \hat{\mathbf{n}}_{2}\right]$ : these are rotation matrices to the mobile bases $\left\{\hat{\boldsymbol{\rho}}_{i}, \hat{\mathbf{v}}_{i}, \hat{\mathbf{n}}_{i}\right\}, i=1,2$. Let $R_{\phi} \hat{\mathbf{e}}$ denote the rotation of an angle $\phi$ around the unit vector $\hat{\mathbf{e}}$. Then $R_{\eta_{1} \delta t \hat{\mathbf{n}}_{1}}=$ $V_{1} R_{\eta_{1} \delta t \hat{\mathbf{z}}} V_{1}^{T}$ ( $\hat{\mathbf{z}}$ is the third unit vector of the reference frame defining our rectangular coordinates) is the parallel transport matrix along the geodesic on the unit sphere defined by $\mathcal{A}_{1}$ to time $\bar{t}_{2}$; hence $\hat{\boldsymbol{\rho}}_{12}=R_{\eta_{1} \delta t} \hat{\mathbf{n}}_{1} \hat{\boldsymbol{\rho}}_{1}$ is the predicted observation direction at time $\bar{t}_{2}$, assuming the trajectory is a great circle and the proper motion is constant. By exchanging the order of the two attributables we can compute $R_{-\eta_{2} \delta t \hat{\mathbf{n}}_{2}}=V_{2} R_{-\eta_{2} \delta t \hat{\mathbf{z}}} V_{2}^{T}$ and $\hat{\boldsymbol{\rho}}_{21}=R_{-\eta_{2} \delta t \hat{\mathbf{n}}_{2}} \hat{\boldsymbol{\rho}}_{2}$, that is the prediction at time $\bar{t}_{1}$. We use the metric

$$
d\left(\hat{\boldsymbol{\rho}}_{1}, \hat{\boldsymbol{\rho}}_{2}\right)=\min \left\{\widehat{\hat{\boldsymbol{\rho}}_{12}, \hat{\boldsymbol{\rho}}_{2}}, \widehat{\hat{\boldsymbol{\rho}}_{21}, \hat{\boldsymbol{\rho}}_{1}}\right\}
$$

that is the minimum between the two angular differences, discarding pairs of attributables that give rise to a large value of this metric.

Note that the proper motion does not vary too much in the time interval between the two attributables provided $\delta t_{\max }$ is small enough; thus, if we want to use large values of $\delta t$, we have also to allow large values of the metric $d$.

\subsubsection{Second filter: symmetric LLS fit.}

Given the two attributables $\mathcal{A}_{1}, \mathcal{A}_{2}$ at times $\bar{t}_{1}, \bar{t}_{2}$ we perform a quadratic approximation of the apparent motion on the celestial sphere $S^{2}$ by using a Linear Least Squares (LLS) fit. The apparent motion is given by the functions $\alpha(t), \delta(t)$. We approximate $\alpha(t), \delta(t)$ with second degree polynomials whose coefficients are derived from a least squares fit. We denote the approximating quadratic functions as

$$
\alpha(t)=\alpha_{q}+\dot{\alpha}_{q}(t-\bar{t})+\frac{1}{2} \ddot{\alpha}_{q}(t-\bar{t})^{2}, \quad \delta(t)=\delta_{q}+\dot{\delta}_{q}(t-\bar{t})+\frac{1}{2} \ddot{\delta}_{q}(t-\bar{t})^{2},
$$

where $\bar{t}=\frac{1}{2}\left(\bar{t}_{1}+\bar{t}_{2}\right)$ is the mean of the times of the attributables. The corresponding time derivatives are

$$
\dot{\alpha}(t)=\dot{\alpha}_{q}+\ddot{\alpha}_{q}(t-\bar{t}), \quad \dot{\delta}(t)=\dot{\delta}_{q}+\ddot{\delta}_{q}(t-\bar{t})
$$

We want to determine the 6 quantities $\alpha_{q}, \dot{\alpha}_{q}, \ddot{\alpha}_{q}, \delta_{q}, \dot{\delta}_{q}, \ddot{\delta}_{q}$ using the data coming from the attributables. The vector of residuals is

$$
\boldsymbol{\xi}=\left(\mathcal{A}_{1}-\mathcal{A}\left(\bar{t}_{1}\right), \mathcal{A}_{2}-\mathcal{A}\left(\bar{t}_{2}\right)\right)^{T},
$$


with $\mathcal{A}(t)=(\alpha(t), \delta(t), \dot{\alpha}(t), \dot{\delta}(t))$.

Given the covariance matrices $\Gamma_{\mathcal{A}_{1}}, \Gamma_{\mathcal{A}_{2}}$ associated to the attributables we use them to weight the residuals in the definition of the target function:

$$
Q(\boldsymbol{\xi})=\frac{1}{8} \boldsymbol{\xi} \cdot W \boldsymbol{\xi}, \quad \text { where } \quad W^{-1}=\left(\begin{array}{cc}
\Gamma_{\mathcal{A}_{1}} & 0 \\
0 & \Gamma_{\mathcal{A}_{2}}
\end{array}\right) .
$$

We introduce the notation

$$
\mathbf{x}=\left(\alpha_{q}, \dot{\alpha}_{q}, \ddot{\alpha}_{q}, \delta_{q}, \dot{\delta}_{q}, \ddot{\delta}_{q}\right)^{T}, \quad \vec{\lambda}=\left(\mathcal{A}_{1}, \mathcal{A}_{2}\right)^{T} .
$$

The value of $\boldsymbol{\xi}=\boldsymbol{\xi}(\mathbf{x})$ that minimizes the target function is obtained by solving the normal equation

$$
C \mathbf{x}=-B^{T} W \vec{\lambda}, \quad \text { where } B=\frac{\partial \boldsymbol{\xi}}{\partial \mathbf{x}}, \quad C=B^{T} W B,
$$

and the matrix $B$ has the form

$B=-\left(\begin{array}{c}B_{1} \\ B_{2}\end{array}\right) \quad$ with $\quad B_{i}=\left(\begin{array}{cccccc}1 & \left(\bar{t}_{i}-\bar{t}\right) & \frac{1}{2}\left(\bar{t}_{i}-\bar{t}\right)^{2} & 0 & 0 & 0 \\ 0 & 0 & 0 & 1 & \left(\bar{t}_{i}-\bar{t}\right) & \frac{1}{2}\left(\bar{t}_{i}-\bar{t}\right)^{2} \\ 0 & 1 & \left(\bar{t}_{i}-\bar{t}\right) & 0 & 0 & 0 \\ 0 & 0 & 0 & 0 & 1 & \left(\bar{t}_{i}-\bar{t}\right)\end{array}\right)$

for $i=1,2$. Once the value of $\mathbf{x}$ is given, we compute the residuals $\boldsymbol{\xi}(\mathbf{x})$ and use the norm $\sqrt{Q(\boldsymbol{\xi})}$ to decide which are the pairs of attributables $\left(\mathcal{A}_{1}, \mathcal{A}_{2}\right)$ to discard. We also discard the pairs giving rise to a large value of the quantity

$$
\kappa_{q} \eta_{q}^{2}=\frac{1}{\eta_{q}}\left[\left(\ddot{\delta}_{q} \dot{\alpha}_{q}-\ddot{\alpha}_{q} \dot{\delta}_{q}\right) \cos \delta_{q}+\dot{\alpha}_{q}\left(\eta_{q}^{2}+\dot{\delta}_{q}^{2}\right) \sin \delta_{q}\right],
$$

where $\eta_{q}=\sqrt{\dot{\delta}_{q}^{2}+\dot{\alpha}_{q}^{2} \cos ^{2} \delta_{q}}$ and $\kappa_{q}$ is the geodesic curvature (see [16], Chapter 9 ).

\subsection{Results}

The accuracy of the linkage method can be measured by the number of true identifications over the total number of identifications found. This computation includes duplications due to alternative solutions. The total number is 3906 and the true ones (that may be related to the same object if it has more than 2 tracklets) are 3144 , i.e. $80.5 \%$ of the total. We could eliminate almost half of the 762 false identifications by lowering from 0.15 to 0.06 arcsec the control on the RMS for acceptable orbits after differential corrections: but this would make us lose 102 true identifications.

In Table 3 we show the efficiency of the linkage procedure, that is we write the number of objects for which at least a pair of tracklets has been correctly linked, giving the details for the MB (Main Belt) and the NEO (Near Earth Object) class. As expected, the efficiency appears greater if there are three tracklets that can be pairwise linked. We stress that we have tested our method with data for which the other available methods in [13], [7] could not perform the linkage. 


\begin{tabular}{|c|c|r|r|r|r|}
\hline with 2 tracklets in 2 nights & Total & \multicolumn{2}{|c|}{ Found } & \multicolumn{2}{|c|}{ Lost } \\
\hline all & 1074 & 963 & $89.7 \%$ & 111 & $10.3 \%$ \\
MB & 1038 & 947 & $91.2 \%$ & 91 & $8.8 \%$ \\
NEO & 19 & 9 & $47.4 \%$ & 10 & $52.6 \%$ \\
\hline \hline with 3 tracklets in 3 nights & Total & \multicolumn{2}{|c|}{ Found } & \multicolumn{2}{|c|}{ Lost } \\
\hline All & 214 & 205 & $95.8 \%$ & 9 & $4.2 \%$ \\
MB & 197 & 196 & $99.5 \%$ & 1 & $0.5 \%$ \\
NEO & 3 & 2 & $66.7 \%$ & 1 & $33.3 \%$ \\
\hline
\end{tabular}

Table 3: Efficiency of the identification procedure.

\section{Conclusions and future work}

We have investigated an orbit determination method that is based on the integrals of the Kepler problem and is suitable to be used with modern data sets of observations. With the recent technologies we can collect a large number of tracklets in each observing night and it is difficult even to relate two tracklets of different nights as belonging to the same observed object. We have defined a linkage procedure between tracklets, allowing to compute preliminary orbits with covariance matrices. An interesting feature that comes out from our numerical experiments is that this method appears to work also when the time span between the two attributables is large, hence it can be used in cases where other linkage methods fail. The efficiency and performance of the algorithm explained in Subsection 4.1 have been studied with a large scale test. Therefore this method can be important for two kinds of applications: 1) to recover objects whose orbit could not be computed with either the classical or the modern known algorithms; 2) to design the scheduler of new surveys planning a smaller number of observations for each object.

The number of alternative solutions of the problem deserves a deeper investigation, however we expect that the acceptable ones should often be much less than 48, the total degree of the polynomial system (9). Moreover the performance of the second algorithm to solve (9), described in Subsection 4.2, has not been tested yet: we would like to perform further experiments to decide if it allows to decrease the computation time.

\section{Acknowledgements}

We wish to thank Massimo Caboara, from the University of Pisa, for his useful suggestions on the algebraic aspects of this work. We are also grateful to Robert Jedicke and Larry Dennau, from the MOPS team of the Pan-STARRS project, for providing us with the simulation data used in Section 7 . Finally we would like to thank Jean-Marc Petit, whose comments have allowed us to improve the manuscript. 


\section{References}

[1] Bate, R. R., Mueller, D. D. and White, J. E.: 1971, Fundamentals of Astrodynamics, Dover publications.

[2] Bini, D. A.: 1997, Numerical computation of polynomial zeros by means of Aberth method, Numer. Algorithms, 13, no. 3-4, 179-200.

[3] Cox, D. A., Little, J. B., O'Shea, D.: 1996, Ideals, Varieties and Algorithms, Springer.

[4] Gauss, C. F.: 1809, Theoria motus corporum coelestium in sectionibus conicis solem ambientium, reprinted by Dover publications (1963).

[5] Gronchi, G. F.: 2002, On the stationary points of the squared distance between two ellipses with a common focus, SIAM Journ. Sci. Comp. 24, no. $1,61-80$.

[6] Gronchi, G. F.: 2009, Multiple solutions in preliminary orbit determination from three observations, CMDA 103, no.4, 301-326.

[7] Kubica, J., Denneau, L., Grav, T., Heasley, J., Jedicke, R., Masiero, J., Milani, A., Moore, A., Tholen, D. and Wainscoat, R. J.: 2007, Efficient intra- and inter-night linking of asteroid detections using kd-trees, Icarus 189, 151-168.

[8] Laplace, P. S.: 1780, Mém. Acad. R. Sci. Paris, in Laplace's collected works 10, 93-146.

[9] Leuschner, A.O.: 1912, On the Laplacean orbit methods, Proceedings of the ICM, 209-217.

[10] Merton, G.: 1925, A modification of Gauss's Method for the Determination of Orbits, MNRAS 85, 693-731

[11] Milani, A., Sansaturio, M. E., Chesley, S. R.: 2001, The Asteroid Identification Problem IV: Attributions, Icarus 151, 150-159.

[12] Milani, A., Gronchi, G. F., de’Michieli Vitturi, M., Knežević, Z.: 2004, Orbit Determination with Very Short Arcs I. Admissible Regions, CMDA 90, 59-87.

[13] Milani, A., Gronchi, G. F., Knežević, Z., Sansaturio, M. E., Arratia, O.: 2005, Orbit Determination with Very Short Arcs II. Identifications, Icarus 79, 350-374.

[14] Milani, A., Gronchi, G. F., Knežević, Z. : 2007, New Definition of Discovery for Solar System Objects, Earth Moon Planets 100, 83-116.

[15] Milani, A., Gronchi, G. F., Farnocchia, D., Knežević, Z., Jedicke, R., Denneau, L. and Pierfederici, F.: 2008, Topocentric Orbit Determination: algorithms for the next generation surveys, Icarus 195, 474-492. 
[16] Milani, A., Gronchi, G. F.: 2009, Theory of Orbit Determination, Cambridge University Press

[17] Plummer, H. C.: 1918, An introductory treatise on Dynamical Astronomy, Cambridge University press, reprinted by Dover publications, New York (1960).

[18] Poincaré, H.: 1906, Sur la détermination des orbites par la méthode de Laplace, Bulletin astronomique 23, 161-187.

[19] Taff, L. G., Hall, D. L.: 1977, The use of angles and angular rates. I Initial orbit determination, CMDA 16, 481-488

[20] Taff, L. G., Hall, D. L.: 1980, The use of angles and angular rates. II Multiple Observation Initial orbit determination, CMDA 21, 281-290 